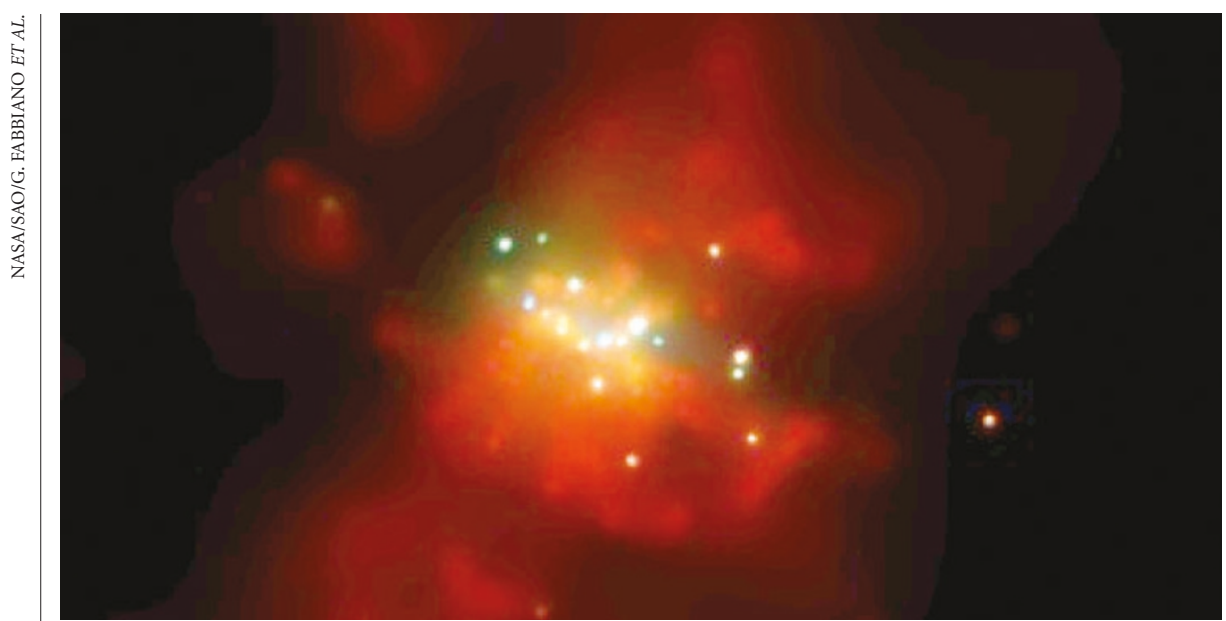

Figure 1 Bright lights. Captured in this false-colour image from the Chandra X-ray Observatory is a population of extremely bright, point-like sources in the galaxy M82, 11 million light years from Earth. Their 'ultraluminous' emission might result from the accretion of matter by intermediate-mass black holes. Dynamical simulations by Portegies Zwart et al. ${ }^{2}$ suggest that in at least one star cluster in the galaxy - MGG 11, which coincides with one of the ultraluminous sources - collisions between stars could have created a 'runaway' star that ultimately became an intermediate-mass black hole.

The authors' simulations indicate that, in this cluster, a runaway star could form on the necessary timescale. The result is tantalizing — this could well be how the building blocks of supermassive black holes formed.

Deep-space images from the Hubble Space Telescope indicate that galaxy mergers were common at early times in the history of the Universe, and the conditions in the centres of those merging galaxies might have resembled those seen in nearby starburst galaxies today. The link between ULXs and intermediate-mass black holes is, however, not conclusive. There are plausible alternative explanations for the high X-ray luminosities of ULXs, such as beaming ${ }^{10}$ (the observed X-ray flux is emitted through a narrow opening angle rather than isotropically) and super-Eddington emission (attributed to 'clumpy' accretion disks ${ }^{11}$ ). More high-resolution observations of super star clusters and ULXs, coupled with simula- tions of star-cluster dynamics, will ensure that the debate over intermediate-mass black holes continues.

Nate McCrady is in the Department of Astronomy,

University of California, Berkeley,

California 94703, USA.

e-mail:nmccrady@astro.berkeley.edu

1. Ghez, A. M., Morris, M., Becklin, E. E., Tanner, A. \& Kremenek, T. Nature 407, 349-351 (2000).

2. Portegies Zwart, S. F., Baumgardt, H., Hut, P., Makino, J. \& McMillan, S. L. W. Nature 428, 724-726 (2004)

Kaaret, P. et al. Mon. Not. R. Astron. Soc. 348, L28-L32 (2004). 1035-1043 (2001).

. van der Marel, R. P. in Coevolution of Black Holes and Galaxies (ed. Ho, L. C.) (Cambridge Univ. Press, in the press).

6. Gurkan, M. A., Freitag, M. \& Rasio, F. A. Astrophys. J. 604, 632-652 (2004).

. Bonnell, I. A. \& Bate, M. R. Mon. Not. R. Astron. Soc. 336, 659-669 (2002).

8. McCrady, N., Gilbert, A. M. \& Graham, J. R. Astrophys J. 597, 240-252 (2003).

9. Kaaret, P. et al. Mon. Not. R. Astron. Soc. 321, L29-L31 (2001).

10. King, A. R., Davies, M. B., Ward, M. J., Fabbiano, G. \& Elvis, M. Astrophys. J. 552, L109-L112 (2001).

11. Begelman, M. C. Astrophys. J. 551, 897-906 (2001).
4. Fabbiano, G., Zezas, A. \& Murray, S. S. Astrophys. J. 554,

Cancer

\title{
Kip moving
}

\author{
John G. Collard
}

The $\mathrm{p} 27^{\mathrm{Kip} 1}$ protein inhibits cell proliferation, helping to prevent tumours developing. We now know that it also affects cell migration, by regulating Rho proteins. Does this function influence tumour progression?

T umours arise when cells proliferate in an uncontrolled manner and, later, take on malignant characteristics, such as the ability to invade surrounding tissues. Particularly aggressive tumours can also metastasize - they spread to distant organs. These changes in tumour-cell properties are driven by alterations that favour various steps in the process: for instance, lower concentrations of the tumour-suppressor protein $\mathrm{p} 27^{\mathrm{Kip} 1}$ lead to deregulated cell proliferation and so are thought to promote tumour formation. There are hints that this protein might also control other stages of cancer development; notably, it regulates cell migration - a prerequisite for metastasis. Writing in Genes and Development, Besson et al. ${ }^{1}$ suggest how it does so.

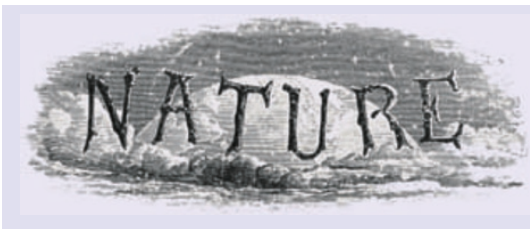

100 YEARS AGO

The administration of chloroform is a subject that is of personal and direct interest to everyone in this present age of civilisation. Sooner or later either we ourselves or those dear to us gladly accept the relief from suffering that is offered, and that chloroform shall be given so that no unavoidable risk is run is a necessity that forces itself on our attention. That much remains to be done in the direction of safety is only too evident. We confess to perusing the diagram of the yearly increasing deathrate from chloroform... with a feeling of horror, and that is deepened when we read the instances given of such deaths, and supplemented by others which have come to our knowledge independently, where chloroform has been given for a trifling operation to an otherwise healthy patient, and where the phrase "Death from cardiac syncope" has acted as an anaesthetic to the conscience of the ignorant and careless anaesthetist.

From Nature 14 April 1904.

\section{YEARS AGO}

High Altitude Rocket Research. Men have long been attracted by the possibility of being able to carry out explorations far above the earth's surface. In 1784 Vincent Lunardi made the first balloon ascent from London, his achievement arousing great popular enthusiasm. Attention, too, was directed to the advances in knowledge which might be gained... When rather more than a century and a half later rockets began to fall on London they aroused different feelings; but men of science found some compensation in the recognition of the fact that a weapon of real use had been developed. During the early part of 1946, an important meeting was held in Washington, D.C., at which the exploitation of the German invention was discussed and a programme of fundamental research was formulated... A rapidly moving rocket does not, of course, provide an ideal platform on which to mount measuring instruments; but in spite of this many results have been derived. Perhaps the most valuable are those relating to the density and the temperature of the upper atmosphere, those relating to the electric current system causing the diurnal variation in the magnetic field, and those relating to the solar emission in the spectral range which cannot be observed from ground-level.

From Nature 17 April 1954. 


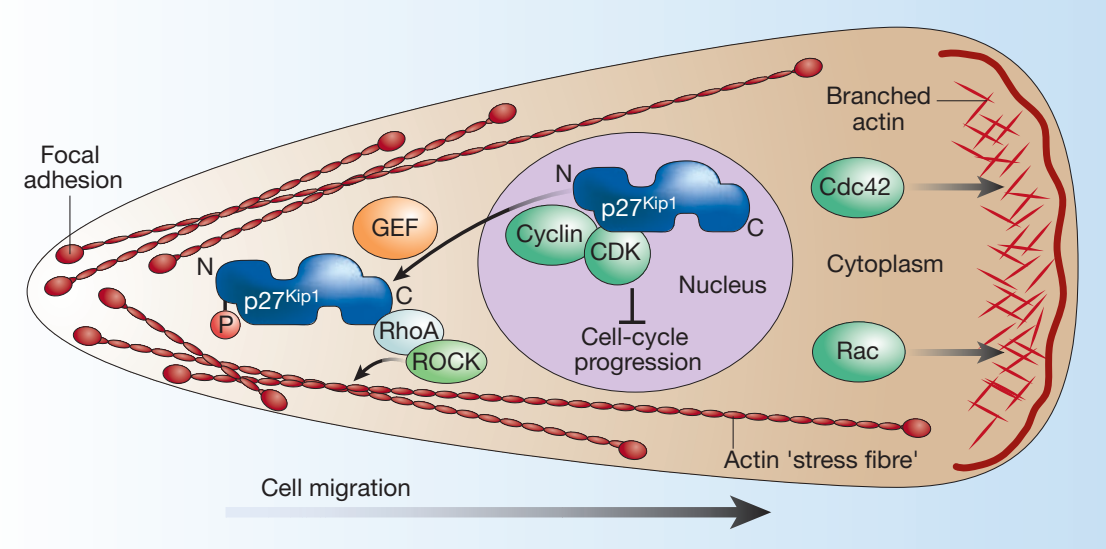

Figure 1 Dual function of a tumour suppressor. In the cell nucleus, the tumour-suppressor protein p2 $7^{\text {Kip1 }}$ binds with its amino-terminal region $(\mathrm{N})$ to complexes of cyclins and cyclin-dependent kinases (CDKs), so inhibiting cell proliferation. When phosphorylated $(\mathrm{P})^{5}, \mathrm{p} 27^{\mathrm{Kip} 1}$ might move into the cytoplasm, where, as shown by Besson et al. ${ }^{1}$, it binds through its carboxy terminus (C) to RhoA and interferes with RhoA activation by guanine-nucleotide-exchange factors (GEFs). RhoA, Cdc42 and Rac regulate the cytoskeletal changes required for cell migration. Cdc42 and Rac work mainly at the front of polarized cells, regulating actin-driven protrusion and the formation of new adhesions required for forward movement. RhoA, through the ROCK protein, works mainly at the rear, determining (among other processes) the turnover of adhesive sites known as focal adhesions and thereby rear retraction. By interfering with RhoA activation, $\mathrm{p} 27^{\mathrm{Kip} 1}$ inhibits or promotes cell migration, depending on the cell type.

Whether or not this property does indeed contribute to cancer progression remains to be seen.

Cell proliferation is controlled by the sequential activation of a group of enzymes called cyclin-dependent kinases (CDKs), which are switched on when bound to cyclin proteins. The $\mathrm{p} 27^{\mathrm{Kip} 1}$ protein inhibits certain cyclin-CDK complexes in the cell nucleus, so preventing proliferation. The relevance of this protein to the control of proliferation is illustrated by the increased body size of p2 $7^{\text {Kipl }}$-deficient mice. Studies of such animals have also revealed that, in general, a decrease or lack of $\mathrm{p} 27^{\mathrm{Kip} 1}$ increases the formation of tumours that are induced by various different oncogenic events, and also increases tumour-associated death rates ${ }^{2,3}$.

In humans, too, abnormally low concentrations of $\mathrm{p} 27^{\mathrm{Kip} 1}$ are often found in tumours ${ }^{4}$. These low concentrations are also associated with tumour aggressiveness and patient mortality - characteristics that are generally related to tumour-cell invasion and metastasis. This raises the question of whether $\mathrm{p} 27^{\mathrm{Kipl}}$ affects these types of tumourcell properties, as well as proliferation. Last year, McAllister et al..$^{5}$ obtained results that suggest this might be true - results that are now supported by Besson and colleagues ${ }^{1}$. In short, these groups have shown that, when found in the cellular cytoplasm, p $27^{\text {Kipl }}$ influences cell migration.

Besson et al. also provide an explanation for this effect on cell migration: they find that $\mathrm{p} 27^{\mathrm{Kipl}}$ binds to, and regulates the activity of, Rho proteins (Fig. 1). Members of the Rho family, which include Cdc42, Rac and RhoA, act as molecular switches in signalling path- ways that affect gene transcription, as well as those that control the filaments of actin proteins in the cell's internal skeleton ${ }^{6}$. In particular, Rho proteins regulate and coordinate the cytoskeletal 'remodelling' that underlies changes in cell adhesion and migration. Rho proteins are switched on when bound to guanosine triphosphate (GTP), and off when bound to guanosine diphosphate (GDP). When active, they can interact with many different effector proteins that elicit different downstream responses. Various other proteins control the on/off state; guanine-nucleotide-exchange factors (GEFs), for instance, activate Rho proteins by promoting the replacement of GDP with GTP. Besson et al. show that $\mathrm{p} 27^{\mathrm{Kip} 1}$ inhibits RhoA activation by interfering with its interaction with GEFs.

The authors further find that the motility of $\mathrm{p} 27^{\mathrm{Kip} 1}$-deficient fibroblast cell types decreases markedly in comparison with that of wild-type cells. Consistent with the earlier study ${ }^{5}$, re-expression of either wild-type $\mathrm{p} 27^{\mathrm{Kip} 1}$ or a truncated mutant that cannot bind to cyclin-CDK complexes restores migration - showing that the protein's regulation of motility is independent of its effects on proliferation. Indeed, the region of $\mathrm{p} 27^{\mathrm{Kip} 1}$ that binds RhoA and is required for migration is different from the region that binds cyclin-CDKs (Fig. 1).

The authors also discover that nonmigratory, p27 $7^{\text {Kipl}}$-deficient cells contain more actin stress fibres and focal adhesions - cellular features that are indicative of high RhoA activity - than wild-type cells. This could be confirmed by testing RhoA activity in cells. Interestingly, inhibiting the enzyme
ROCK, a downstream effector of RhoA, restores the migration of $\mathrm{p} 27^{\mathrm{Kip} 1}$-deficient cells in response to growth factors. Apparently, then, in fibroblasts a decrease in $\mathrm{p} 27^{\text {Kip } 1}$ concentration increases RhoA activity, which in turn increases ROCK activity, thereby hampering migration. Dampening Rho signalling, as occurs with normal p $27^{\text {Kip1 }}$ concentrations or ROCK inhibition, allows fibroblasts to migrate again.

For tumours to metastasize, cells must alter their connections to their neighbours and their substrate, and then migrate. So could the effects of $\mathrm{p} 27^{\text {Kip } 1}$ on migration somehow influence tumour progression? This is not easy to say, because models in vitro do not always mirror events in vivo - and different cell types vary in any case. For instance, efficient migration requires a tightly balanced activation and deactivation of Cdc42, Rac and RhoA in both space and time $^{6}$. Low RhoA activity might inhibit migration by preventing cells from achieving sufficiently strong adhesion, and hence traction, to move forward. But excessive RhoA activity can also inhibit migration, by gluing cells to their substrate too strongly. Increased Rac-mediated cell-to-cell interactions also prevent migration ${ }^{7}$.

Still more complexity has been observed in a three-dimensional matrix ${ }^{8}$ in which cells can move in either an amoeboid or an elongated mode. Amoeboid migration is dependent on RhoA and ROCK, whereas the elongated mode is associated with Racdependent protrusions that are rich in actin filaments ${ }^{9}$. The degree of cell migration therefore depends on both the conditions and the cell type. So it is not surprising that the effects of $\mathrm{p} 27^{\mathrm{Kip} 1}$ on migration are also cell-type-dependent. For instance, overexpression of $\mathrm{p} 27^{\mathrm{Kip} 1}$ stimulates the migration of liver-cancer cells and fibroblasts ${ }^{1,5}$ but inhibits the migration of some other cell types, such as endothelial cells ${ }^{10}$. Consequently, aberrant $\mathrm{p} 27^{\mathrm{Kipl}}$ signalling might promote the invasiveness and malignancy of some tumours but inhibit that of others. In support of this, a loss of $\mathrm{p} 27^{\text {Kipl }}$ usually correlates with increased tumour aggressiveness and a poor clinical outcome ${ }^{4}$. But in some tumour types, high $\mathrm{p} 27^{\text {Kipl }}$ concentrations do so ${ }^{11,12}$.

One source of cell-type variability might lie in the fact that $\mathrm{p} 27^{\mathrm{Kip} 1}$ can regulate Rho activity only when $\mathrm{p} 27^{\mathrm{Kip} 1}$ is localized in the cytoplasm $^{1,5}$. Unlike other tumour suppressors, $\mathrm{p} 27^{\mathrm{Kip} 1}$ activity is downregulated by increased degradation or by exclusion from the nucleus. If the protein's concentration remains high but it is excluded from the nucleus, it can no longer regulate the cell cycle (so leading to increased proliferation), yet can interfere with RhoA and thereby influence cell migration and tumour progression. If its concentration is lowered but it remains in the nucleus, however, it again 
cannot dampen cell proliferation, but neither can it influence migration.

Another confounding factor is that Rho proteins control a wide range of signalling pathways, which, when deregulated, can result in various cellular abnormalities ${ }^{13}$. As well as influencing migration, these proteins might affect cell survival, growth and differentiation $^{6}$, thereby influencing tumour development and progression ${ }^{7}$. You won't be surprised to hear, then, that it will be a challenge to determine whether $\mathrm{p} 27^{\mathrm{Kip} 1}$ contributes to tumour progression in vivo via regulation of the cell cycle, or Rho-mediated signalling, or both. The answer awaits the generation of mice in which wild-type $\mathrm{p} 27^{\mathrm{Kip} 1}$ is replaced by a mutant version that lacks the binding domain for either cyclin-CDK complexes or RhoA. All being well, these studies will reveal the true role of $\mathrm{p} 27^{\mathrm{Kip} 1}$ in cancer.

John G. Collard is at the Netherlands Cancer

Institute, Division of Cell Biology, Plesmanlaan 121, 1066 CX Amsterdam, The Netherlands.

e-mail: j.collard@nki.nl

1. Besson, A., Gurian-West, M., Schmidt, A., Hall, A. \& Roberts, J. M. Genes Dev. 18, 862-876 (2004).

2. Philipp-Staheli, J., Payne, S. R. \& Kemp, C. J. Exp. Cell Res. 264, 148-168 (2001).

3. Philipp-Staheli, J. et al. Cancer Cell 1, 355-368 (2002).

4. Slingerland, J. \& Pagano, M. J. Cell. Physiol. 183, 10-17 (2000).

5. McAllister, S. S., Becker-Hapak, M., Pintucci, G., Pagano, M. \& Dowdy, S. F. Mol. Cell. Biol. 23, 216-228 (2003).

6. Ridley, A. J. et al. Science 302, 1704-1709 (2003).

7. Malliri, A. \& Collard, J. G. Curr. Opin. Cell Biol. 15, 583-589 (2003).

8. Friedl, P. \& Wolf, K. Nature Rev. Cancer 3, 362-374 (2003).

9. Sahai, E. \& Marshall, C. J. Nature Cell Biol. 5, 711-719 (2003).

10. Goukassian, D. et al. FASEB J. 15, 1877-1885 (2001).

11. Kouvaraki, M. et al. Cancer 94, 2454-2465 (2002).

12. Saez, A. et al. Br. J. Cancer 80, 1427-1434 (1999).

13. Boettner, B. \& van Aelst, L. Gene 286, 155-174 (2002).

Animal behaviour

\section{Fickle females?}

Michael J. Ryan

The courtship of satin bowerbirds is a complicated business. Different parts of a male's display appeal to females of different ages, so age-biased variation might underlie the evolution of these displays.

A common complaint among courting men is that women do not know what they want. Virgil, for instance, observed that "A woman is always a fickle, unstable thing". How, then, can a male cope? One solution would be to assess the general preference in this sea of shifting female wants and desires, and bias courtship to the majority opinion. Another would be to appeal simultaneously to multiple preferences, to be something to everyone. This second solution, we are told by Coleman and colleagues on page 742 of this issue ${ }^{1}$, is the one adopted by male satin bowerbirds (Ptilonorhynchusviolaceus).

These males have a complex set of courtship rituals. They decorate their bowers with blue objects, and they also combine a suite of vocalizations and movements into a behavioural display directed at females. Another layer of complexity derives from the timing of these display components. In the first stage of courtship a male is not present at the bower but his decorations are. Thus, the only way for a female to evaluate the male is through this evidence. In the second stage, a female returns to a subset of the original bowers she sampled. During this stage, both bower decorations and the male, who intensely courts the female, are present (Fig. 1), and the female builds nests at several bowers. In the final stage, the female makes several visits to where she constructed nests and decides on a single mate.

Coleman and colleagues' experiments ${ }^{1}$ were underpinned by an observation from

a study ${ }^{2}$ by members of the same group. Younger females, it turned out, are more threatened by the physical male displays than are older females. Could variation in this female fear factor generate selection for complex displays in males? In experiments conducted over a two-year period, Coleman et al. addressed the question by augmenting some bowers with extra decorations (treatment bowers) while leaving others unmanipulated (control bowers). Using video recordings, they determined the effect of bower treatment on the frequency of visits by females of different ages - first year, second year and three-plus years - at each of the three stages of courtship.

Of the bowers sampled during the first stage of courtship, all females were more likely to return to treated rather than control bowers in the second stage (remember, males are not present during the first stage). The same was not true, however, when females moved from the second stage, when the male is present and subjects the females to intense displays, to the third stage. Whereas younger females were more likely to visit the experimental than control bowers in the third stage, augmented bower decorations had no such effect on the behaviour of older females.

During the final stage of courtship, females decide on a mate. Here again there is an age-biased effect of bower treatment. In both years of Coleman and colleagues' study, first-year females preferred to mate with males in more decorated bowers, but the treatment did not influence the mate choice of the three-plus-year females. In year 1 of the study, the second-year females' choice of mate showed no effect of bower treatment, but they did in year 2, when even more decorations were added.

An age-biased effect is not restricted to female responses to bower decorations, but also applies to male display intensity. The more intense the male display during the second stage of courtship, the more likely were older females to visit a male in the third stage. But that did not apply to the two younger cohorts. Coleman et al. ${ }^{1}$ conclude that selection generated by age-biased variation in female choice is responsible for the evolution of complex courtship in these bowerbirds. The fear that the male's display instils in younger females seems to drive the age-biased differences in courtship preference.

Clearly, the further elaboration of bower decorations to the degree used in the manipulations would be under stronger selection from younger rather than older females. In this study, however, it is not clear if older females would generate selection to maintain the current level of bower decorations. Perhaps there is a lower threshold for this component of the display in older than in younger females; if so, older females alone could generate the selection needed to maintain this complex display. The fact that second-year females were not influenced by augmented bower decorations in year 1 of the study, but were in year 2 when more decorations were used, suggests that thresholds for an effect might change continuously with age. This age class could also offer a target group for research to understand the relative value of decorations and male display intensity for females of different ages.

A larger question concerns the evolutionary pattern of bowerbird displays. The complexity of bowers, decorations and courtship behaviour varies greatly among different species of bowerbird ${ }^{3,4}$. Can these broad macroevolutionary patterns among species be explained by the same microevolutionary process suggested by the findings of Coleman et al. ${ }^{1}$ ?

Studies of fish ${ }^{5}$, frogs ${ }^{6}$, cockroaches ${ }^{7}$ and humans ${ }^{8}$ have shown that female mating preferences can change as a function of female age, size and reproductive status. Such variation in preferences can contribute to the maintenance of variation in male traits. Young female guppies, for example, prefer males with more orange pigmentation, whereas older females are agnostic about colour and seem to prefer courtship vigour ${ }^{5}$; larger female cricket frogs prefer lower-frequency mating calls, but smaller females prefer higher-frequency calls ${ }^{6}$. What is unusual in the case of satin bowerbirds is that the variation in female mating preferences more clearly contributes to the evolution of multi-component and 